DOI: $10.17951 / \mathrm{ff} .2018 .36 .1 .139-150$

\begin{tabular}{lcr}
\hline \multicolumn{3}{c}{ A N N A L ES } \\
UNIVERSITATIS M A R I A E C URIE-SKŁ ODOWS K A \\
LOL. XXXVI & SECTIOFF & $1-2018$ \\
\hline
\end{tabular}

CHRISTINE MARTINEZ

ORCID ID: https://orcid.org/0000-0001-5847-3933

Uniwersytet Warszawski

\title{
Une approche sémantico-pragmatique des collocations du champ lexical écologie en français et leurs équivalences polonaises
}

Semantyczno-pragmatyczna analiza kolokacji w polu leksykalnym écologie (ekologia) w języku francuskim i ich polskie odpowiedniki

A semantic-pragmatic analysis of collocations of the lexical field ecology in French and their Polish equivalences

\section{INTRODUCTION}

L'écologie est un domaine actuel qui présente de nombreuses unités lexicales, c'est pourquoi il nous semble nécessaire de les analyser pour éviter les confusions. Nous souhaitons présenter certaines collocations ainsi que le profil de ces expressions lexicalisées employées au quotidien dans les médias français. Une approche sémantico-discursive de ces collocations (Sablayrolles, 2000, 2011; Sablayrolles et Mejri, 2011; Tutin et Grossmann, 2002; Tutin, 2005), nous permet de créer un profil nommé lexico-discursif par Marie Veniard (2013).

Nous nous restreindrons au niveau sémantico-pragmatique du profil, car c'est celui-ci qui «traduit et conditionne l'expérience qu'une communauté de locuteurs fait d'un objet du monde» (Veniard, 2013: 55). Sachant que ce profil est un aspect de l'analyse du discours particulier à la France, et immanent des corpus français/ francophone, nous essayerons de voir si le profil de certaines collocations ancrées dans les médias français a ses équivalents en polonais.

Pour cet article, nous verrons donc la notion du profil lexico-discursif de Marie Veniard (2013). Puis, une interprétation des exemples français et des quasi-équi- 
valents en polonais sera présentée, et enfin, nos remarques en guise de conclusion cloront ce texte.

\subsection{Définition du profil selon Marie Veniard}

Marie Veniard (2013 : 55) propose de réaliser un profil lexico-discursif de chaque item étudié pour éviter toute mauvaise interprétation ou compréhension d'un énoncé car celui-ci

[...] rend compte des déterminations sémantico-discursives s'exerçant sur le sens du mot. Le profil rassemble les caractéristiques préférentielles de la combinatoire et du fonctionnement discursif de ce mot sur les plans sémantique, syntaxique, syntagmatique (cooccurrence, collocations, cotexte), textuel, énonciatif et interdiscursif.

Le profil lexico-discursif permet aussi de saisir le lexique en discours et également d'apprécier les apports de sèmes. Nous verrons que la création d'un profil lexico-discursif sert à faire ressortir la naissance de sème inhérent au contexte, à l'environnement dans lequel se trouve l'item, qu'il se charge de ce/s sème/s tout en gardant son référent initial, qu'il adopte une particularité sémantico-référentielle qui lui permet de fonctionner à un moment discursif précis. En outre, le profil joue un rôle important dans l'analyse du discours puisqu'au niveau sémantico-pragmatique, comme vu supra, il «traduit et conditionne l'expérience qu'une communauté de locuteurs fait d'un objet du discours » (Veniard, 2013), c'est pour ces raisons que nous nous restreindrons au niveau sémantico-pragmatique du profil.

Le corpus de Marie Veniard était constitué de deux événements de nature conflictuelle: un conflit militaire et un conflit social, dont les mots clés étaient respectivement: Afghanistan et intermittents. Notre étude se limite aux expressions de l'écologie telles que: tourisme écologique - turystyka ekologiczna, et écologie urbaine-ekologia miejska1.

Nous proposons de voir la combinatoire ainsi que les fonctionnements discursifs préférentiels de certains mots; Veniard (2013: 162) déclare en parlant de son profil que "c'est une version plus aboutie de la notion d'habitus discursif»", et qu'une meilleure appréhension du fonctionnement du mot et de son usage est possible grâce à celui-ci.

${ }^{1}$ Expressions recueillies dans des quotidiens en ligne (tels que Le Figaro, Le Monde, Le Nouvel $O b s$, entre autres) qui ont été dépouillés non régulièrement, mais selon les besoins de cette étude.

${ }^{2}$ Qu'elle a emprunté à Plantin (2003) qui lui-même l'a adapté de Bourdieu. 


\subsection{Corpus}

Notre corpus, ici restreint, est constitué de moments discursifs, c'est-à-dire du «surgissement dans les médias d'une production discursive intense et diversifiée à propos d'un même fait» (Moirand, 2004: 72). Le corpus se caractérise par son hétérogénéité multiforme i.e. :

- sémiotique $\rightarrow$ dans l'aire de la page du journal: articles, photographie, typographie, mise en page;

- textuelle $\rightarrow$ différents genres;

- énonciative $\rightarrow$ textes présentant des formes variées de discours autre: citations, interviews, slogan, etc.

Ces moments discursifs, tirés des médias, sont à la base d'un sens social dont émerge une construction discursive qui est introduite par le dispositif sémiotique de la page du journal. Le profil sert à rassembler les fonctions d'une expression lexicalisée à différents niveaux afin de construire et/ou d'actualiser son sens social.

\subsection{Le sens social}

Lors de notre analyse et création du profil, nous avons pris en compte les trois caractéristiques citées supra qui se scindent en six sous-caractéristiques, que nous nommons «traits sémiotiques $»^{3}$ comme suit:

- rubrique habituelle $\rightarrow$ ou création d'une rubrique spéciale, lors de la COP 21, Le Figaro avait créé une rubrique consacrée à cet événement.

- Ajout de pré-titre $\rightarrow$ i.e., un syntagme en majuscules qui précède le surtitre, qui est d'ordre plus général.

- Bandeau spécifique $\rightarrow$ qui donne un titre à la page et se substitue à la rubrique habituelle.

- Appellation générale $\rightarrow$ qui témoigne d'une proximité du sujet.

- Reprises;

- Et enfin, la variation référentielle.

Tous ces facteurs soulignent la malléabilité de l'objet du discours. Le discours est en quelque sorte pré-formé par ces contraintes de divers ordres. Marie Veniard (2013: 60) explique que «chaque journal adopte son propre dispositif sémiotique» ${ }^{4}$, que celui-ci est une étape importante dans la construction de sens. Voyons de plus près ce dispositif sémiotique, puis les différents aspects (différentes facettes) des pages prises en compte pour cette étude, en illustrant avec des exemples.

\footnotetext{
${ }^{3}$ Nous guillemetons cette expression, car elle a été créée pour le besoin de l'étude.

${ }^{4}$ Il s'agit de la mise en page, de la rubrique, de la typographie, du type de discours employés.
} 


\section{ANALYSE DU CORPUS}

\subsection{Tourisme écologique vs turystyka ekologiczna}

Pour illustrer notre analyse, nous débuterons par la collocation : tourisme écologique - turystyka ekologiczna, et celles formées sur la base nom + adjectif, constituée d'adjectifs quasi-synonymes, i.e.: durable, vert-trwała/zrównoważona, zielona. Au préalable, il est précieux d'apporter quelques précisions quant au dispositif sémiotique des quotidiens français et polonais, ce qui nous a permis de rassembler les traits sémiotiques et de les comparer afin de créer un sens social et au final un profil. Les comparaisons des collocations françaises et polonaises se feront ad hoc.

- Rubrique habituelle: Évasion qui présente trente-deux articles (sur une période de 2008 à 2017 pour Madame Figaro) en relation avec le tourisme durable, cependant, seulement 8 reprennent notre concept. Néanmoins, ce ne sont que des variations référentielles telles que: week-ends durables, matières durables, développement durable (réitéré), technologie durable, tourisme durable (2), démarches durables, planète durable.

- Absence de pré-titre et bandeau spécifique dans tous les cas.

- Appellation générale: proximité certifiée par l'adjectif durable, ancré dans le discours des locuteurs. Or, l'absence du paradigme tourisme durable est visible uniquement après la lecture/l'étude de l'article.

- Reprises: aucune dans la même rubrique, peu d'article consacré strictement au tourisme durable.

Le dispositif sémiotique d'un quotidien polonais ressemble-t-il à celui du journal français.

Avec turystyka ekologiczna, le dispositif sémiotique de la page et/ou des pages prises en compte pour cette étude se présentent de la sorte:

- Aucune rubrique spéciale présente les dix-neuf articles (sur une période de 2004 à 2017 pour Wysokie Obcasy de Gazeta Wyborcza) qui soi-disant dégagent la notion de tourisme durable/écologique, néanmoins, aucun ne reprend ce concept. Pour les articles corrélant, ce ne sont que des variations référentielles telles que : eko - éco, ekologiczne gospodarstwo - agriculture écologique, surowiec ekologiczny - matière première écologique, ekologiczna moda - mode écologique, ekologiczny ogród - jardin écologique, myślenie ekologiczne - pensée écologique, świadomość ekologiczna - conscience écologique, ruchy surrealistyczno-ekologiczne - courant surréalistico-écologique, certifikat ekologiczny - certificat écologique.

- Présence de pré-titre du genre: artykut ou tekst z numeru, article en ligne ou texte extrait du numéro (version papier), mais absence de bandeaux spécifiques dans la plupart des cas. 
- Appellation générale: manque total de proximité, l'adjectif trwaty est automatiquement remplacé par ekologiczny - écologique, ancré dans le discours des locuteurs polonais et non ambigu. La présence du syntagme turystyka ekologiczna - tourisme écologique, ou ce que nous appelons ses adaptations sémantico-pragmatiques apparaissent uniquement après la lecture/l'étude de l'article, et sont rares.

- Reprises: aucune reprise et aucun article avec notre syntagme, par conséquent, nous nous sommes retournée vers Gazeta Wyborcza d'où est tirée l'interview présentée.

Les divergences sont palpables, ne serait-ce que par l'absence d'articles dans l'équivalent polonais Wysokie Obcasy. S'il s'agit de l'éventail de propositions des variations préférentielles, il découle du journal lui-même, étant donné qu'un quotidien moins ciblés émet des informations plus générales.

Les dissemblances sont-elles aussi claires in situ? Les extraits suivants sont deux interviews ${ }^{6}$ afin que notre comparaison soit fiable et que le genre journalistique ne fausse nos données et remarques. La première (a) a été relevée dans Madame Figaro (3 avril 2013), la seconde (b) provient de Gazeta Wyborcza (25 avril 2015).

(a) Sur la route du tourisme durable

Tourisme vert, équitable, responsable... Diana Hounslow, lauréate du Trophée Femmes du Tourisme 2013, nous aide à nous y retrouver

Lefigaro.fr/madame. - Entre voyage équitable, responsable ou écolo, y a-t-il une réelle définition du tourisme durable?

Diana Hounslow. - Absolument, l'Organisation mondiale du tourisme exige même le respect de trois règles d'or. La première est d'exploiter de façon optimale les ressources de l'environnement, en préservant les processus écologiques essentiels et en aidant à sauvegarder les ressources naturelles et la biodiversité. La seconde est de respecter l'authenticité socioculturelle des communautés d'accueil. Enfin, il faut assurer une activité économique viable sur le long terme en offrant à toutes les parties prenantes des avantages socio-économiques équitablement répartis, contribuant ainsi à la réduction de la pauvreté.

Concrètement, comment préparer un voyage durable dans le respect de ces trois règles?

Le voyageur responsable sera à la recherche des modes de transport doux et d'hébergements écoresponsables. Il choisira de la même manière les sites à visiter et s'y comportera dans le respect des lieux et de ceux qui y demeurent. Il s'efforcera de pratiquer le tourisme équitable, concentrant ses dépenses sur l'achat de produits fabriqués sur place. Il mangera bio et des productions locales, assurant ainsi des retombées économiques directes sur le territoire visité. Si de nombreux voyageurs sont attirés par les valeurs du tourisme durable, rares sont ceux qui vont jusqu'au bout de ces convictions. [...]

Pourquoi les femmes sont-elles plus réceptives au tourisme durable que les hommes?

D’une part, différentes études liées aux hébergements ont montré que ce sont les femmes, à $60 \%$, qui organisent les voyages. D'autre part, à la maison, ce sont le plus souvent elles qui mettent

${ }^{5}$ Gazeta Wyborcza étant un quotidien présentant des informations de divers domaines sans préciser le sexe du lecteur, tandis que Wysokie Obcasy vise, en général, un public féminin.

${ }^{6}$ Choix intentionnel. 
en place le tri sélectif et choisissent les produits d'entretien. Le choix des aliments ou la qualité de l'eau sont parmi les préoccupations des femmes à travers le monde. Pour elles, les écogestes sont devenus un mode de vie.

(b) Ekoturystyka. Trzeba nauczyć się podróżować bardziej świadomie

Bartłomiej Kuraś: Co to takiego ekoturystyka i turystyka zrównoważona?

Dominika Zaręba: To przeciwieństwo masowej turystyki. Generalnie chodzi o to, by zwiedzając jakiś region, odnajdywać w nim autentyzm, podziwiać przyrodę, przy tym starając się chronić środowisko naturalne. Ekoturystyka, jako najbardziej przyjazna dla środowiska forma turystyki, stanowi rdzeń koncepcji turystyki zrównoważonej. Organizowana jest na terenach cennych przyrodniczo i kulturowo, uczestniczą w niej osoby o dużej świadomości ekologicznej i żądzy poznawczej, a wpływy z niej wspierają bezpośrednio miejscową społeczność i zasilają lokalną gospodarkę. [...]

Właśnie wyruszasz do Ekwadoru na Światowa Konferencję Ekoturystyki i Turystyki Zrównoważonej.

Co tam będziesz robić?

W konferencji w Quito weźmie udział prawie 600 osób z całego świata zaangażowanych w rozwój turystyki oparty na założeniach zrównoważonego rozwoju, reprezentujących branżę turystyczną, instytucje rządowe, organizacje pozarządowe, świat akademicki, media. Motywem przewodnim konferencji jest wzmacnianie zaangażowania sektora turystycznego w realizację rezolucji ONZ właśnie na temat promocji ekoturystyki na rzecz zmniejszania ubóstwa i ochrony środowiska na świecie.

Dominika Zaręba - publicystka, autorka książek i przewodników turystycznych, propagatorka ekopodróżowania.

Ce que nous avons voulu mettre en exergue dans l'exemple (a) sont: en italique les expressions adjacentes à tourisme durable; les mots, expressions soulignés présentent leur emploi concret: respect des règles d'or, préserver les processus écologiques essentiels, aider à sauvegarder les ressources naturelles, la biodiversité, etc. c'est-à-dire tout ce qui est en corrélation avec la notion concrète de l'écologie. Enfin, ce que nous qualifions d'emploi d'identification se manifeste dans les expressions telles que: socio-culturelle, socio-économique, les femmes sont plus réceptives, se comporter, préoccupations des femmes à travers le monde, un mode de vie, par les traits humains intrinsèques émergents.

L'écriture journalistique traduit les différents aspects (facettes) de la notion écologique par:

- les actions : assurer, transport doux, manger bio, tri sélectif, écogestes;

- la dimension politiquement correcte: équitable, respect de trois règles d'or, préservant les processus écologiques essentiels, aidant à sauvegarder les ressources naturelles, la biodiversité, respect des lieux et de locaux, etc.;

- la dimension économique: équitablement réparti, avantages socio-économiques équitablement répartis, la réduction de la pauvreté;

- les citations : lauréate du Trophée Femmes du Tourisme 2013, l'Organisation mondiale du tourisme; 
- les variations référentielles : emploi multiples des quasi-synonymes de tourisme durable ou des pendants de développement durable.

Dans le cas de l'interview polonaise (b), en italique sont marquées les expressions proches et quasi-synonymes de tourisme durable; les mots, expressions soulignés mettent en avant les emplois concrets: Trzeba nauczyć się podróżować - il faut apprendre à voyager, przeciwieństwo masowej turystyki - opposé au tourisme de masse, autentyzm - authenticité, podziwiać przyrodę - admirer la nature, chronić środowisko naturalne - protéger l'environnement, najbardziej przyjazna - la plus douce (respectueuse), cennych - précieux, duża świadomość ekologiczna - une conscience environnementale élevée, wspieraja bezpośrednio miejscowa spoteczność i zasilaja lokalna gospodarke - soutenir la communauté locale et renforcer l'agriculture locale, branżę turystyczna, instytucje rzadowe, organizacje pozarzadowe, świat akademicki, media - le secteur du tourisme, les institutions gouvernementales, les institutions non gouvernementales, le secteur académique et les médias, $O N Z$ - ONU, zmniejszania ubóstwa - la réduction de la pauvreté. Quant à l'emploi d'identification, il point avec nauczyć się bardziej świadomie - apprendre de manière plus consciente, 600 osób z całego świata zaangażowanych - 600 personnes du monde entier engagées/impliquées, propagatorka - propagatrice.

Dans cette interview, l'écriture journalistique polonaise traduit les différents aspects de la notion écologique par:

- les actions: Trzeba nauczyć się bardziej świadomie - Il faut apprendre de manière plus consciente, ou avec les verbes tels que: odnajdywać - retrouver, podziwiać - admirer, chronić - protéger, organizować - organiser, wspierać soutenir, etc.;

- la dimension politiquement correcte: zrównoważonego rozwoju - le développement durable, ochrony środowiska - la protection de l'environnement, et ekopodróżowania - voyage écologique, wspieraja bezpośrednio miejscowa społeczność - soutenir la communauté locale, etc.;

- la dimension économique: przeciwieństwo masowej turystyki-opposé au tourisme de masse, zasilaja lokalna gospodarke - renforcer l'agriculture locale, zmniejszania ubóstwa - la réduction de la pauvreté;

- les citations : Światową Konferencję Ekoturystyki - la conférence mondiale de l'écotourisme, $O N Z$ - ONU;

- les variations référentielles sont réduites à l'usage du quasi-synonyme de tourisme durable: ekoturystyka - écotourisme. Le champ sémantique propre à l'écologie, et plus particulièrement au tourisme écologique, n'est pas autant exploité que dans l'exemple (a), toutefois pour avoir un impact sur le lecteur il y a Światową Konferencję Ekoturystyki - la conférence mondiale de l'écotourisme, 
zrównoważonego rozwoju - le développement durable, ochrony środowiska - la protection de l'environnement, et ekopodróżowania - voyage écologique.

Le discours de l'interview polonaise est plus engagé, plus concret, moins concentré sur le voyage synonyme du bien-être - comme c'est le cas dans l'interview française, il se focalise plus sur le politiquement correct, la subjectivité - qui d'ailleurs clôt l'article - quand on y apprend que : Dominika Zaręba est journaliste, auteur de livres et guides de voyages, et propagatrice de l'écotourisme.

\section{2 Écologie urbaine ${ }^{7}$ vs ekologia miejska}

Après avoir vu en détails les divergences existantes entre les collocations tourisme écologique et turystyka ekologiczne, nous proposons de décortiquer nos deux exemples suivants, l'un tiré du Figaro (rubrique immobilier du 15 avril 2016), l'autre de Gazeta Wyborcza (26 juin 2017). Avant cela, voyons ce que le dispositif sémiotique du quotidien français dévoile.

- Rubrique habituelle: oui et non; en effet, en général, l'écologie urbaine se retrouve plus souvent dans la rubrique Politique, Environnement ou Architecture ${ }^{8}$. Or, cet article apparaît dans la rubrique Immobilier.

- Absence de pré-titre et bandeau spécifique.

- Appellation générale: la proximité est certifiée par les adjectifs durable et écologique, ancré dans le discours des locuteurs. Absence du paradigme écologie urbaine.

- Maintes reprises de construction écologique ou écoconstruction.

Quels traits sémiotiques laisse transparaître le quotidien polonais Gazeta Wyborcza.

- Rubrique habituelle: oui, en général, l'écologie se retrouve plus souvent dans la rubrique Wiadomości - Actualités.

- Absence de pré-titre, par contre un bandeau spécifie la présence d'une vidéo - wideo.

- Appellation générale: la proximité est certifiée par l'adjectif ekologiczne - écologique, ancré dans la mémoire des lecteurs ; toutefois, le paradigme écologie urbaine est absent.

- Reprise de ekologiczne, budynku-budownictwo - construction écologique. Illustrons avec deux articles des quotidiens respectifs.

${ }^{7}$ Ville/milieu citadin qui suit les restrictions écologiques imposées par la Communauté européenne.

${ }^{8}$ D'après nos observations. 


\section{(c) Construire durable avec des matériaux écologiques, c'est possible}

Avec le printemps vient le temps de donner un coup de neuf à sa maison! Des salons de l'éco construction se déroulent ce mois-ci un peu partout en France. L'occasion de découvrir l'art et la manière de construire sa maison avec des matériaux écolos... un pari sur l'avenir!

Après Bonifacio le week-end dernier, c'est désormais à Melgven dans le Finistère que va se tenir le salon de l'écoconstruction ces 16 et 17 avril 2016. Après avoir rencontré un franc succès ces dernières années, les journées de l'écoconstruction rassemblent des professionnels de l'habitat et des particuliers désireux d'en savoir plus sur ce nouveau mode de construction écologique. Les stars de ce rendez-vous? Le bois, la paille, le liège, la ouate de cellulose et les matériaux recyclés, mais aussi l'isolation thermique et le solaire photovoltaïque!

Une alternative technique aux matériaux conventionnels

Aujourd'hui, les bâtiments consomment $40 \%$ de l'énergie mondiale et ils sont à l'origine de $36 \%$ des émissions de $\mathrm{CO}_{2}$. La solution? Des constructions intelligentes, combinant une technologie respectueuse de l'environnement et une approche attentive aux besoins des habitants. Pour les salons de l'écoconstruction, l'objectif est clair: montrer que construire ou rénover son habitat avec des matériaux écologiques est tout à fait possible.

Construire durable, oui mais avec quoi? Grâce à des matériaux d'origine naturelle issus des sols (argile, terre, ...), de l'agriculture, de l'élevage ou encore du recyclage, liège, lin, chanvre, papier, ...). Ces écomatériaux sont une véritable alternative aux matériaux conventionnels et ils présentent l'avantage d'être plus respectueux de l'environnement. D'ailleurs pour les convaincus, ces moyens offrent de bien meilleures performances de confort. C'est notamment le cas de la ouate de cellulose, de la paille et du liège qui permettent d'isoler les murs et les toitures, ou encore du pisé, un système de construction en terre crue très efficace pour l'inertie thermique.

Un projet qui peut être intéressant économiquement

Comme quoi ce qui est écologique peut aussi être économique. Le bilan de l'addition pour ces constructions en matériaux écolos peut être gagnant sur le long terme. Car même si l'achat des matériaux représente un investissement plus coûteux au départ - de 1450 à 1820 par $^{2}$ de surface utile -, ceux-ci se veulent plus stables et ils se dégraderont moins vite face aux risques de tassement ou d'humification. D'ailleurs, construire durable c'est aussi penser local. Chaque région a ses ressources, ses spécificités et son savoir-faire traditionnel: une bonne idée donc pour faire tourner l'économie locale en embauchant des artisans qui maîtrisent les éléments naturels de leur région.

Il faut également savoir qu'en cas de construction ou de rénovation énergétique, le Ministère de l'Environnement, de l'Énergie et de la Mer propose des aides financières comme le cumul de l'éco-prêt à taux zéro et du crédit d'impôt Transition Énergétique.

\section{(d) Geolodzy w nowym, ekologicznym budynku na Kampusie UJ}

Zmniejszą się koszty eksploatacji

To, co jednak w całej inwestycji najważniejsze, to ekologia i inteligentne budownictwo. Nowa siedziba ING UJ jest wyposażona w system zarządzania BMS umożliwiający monitorowanie i zarządzanie wszystkimi urządzeniami znajdującymi się w budynku i jego otoczeniu, jest ogrzewana ciepłem Ziemi, z kolei energia elektryczna w słoneczne dni jest generowana w większości przez instalację fotowoltaiczną znajdującą się na południowo-zachodniej fasadzie budynku.

Zielony dach

Jak podkreśla dr Dziubińska, ekologicznego wizerunku obiektu dopełnia zielony dach budynku. - Posadzona roślinność nie wymaga znacznej ingerencji człowieka, dzięki czemu minimalizuje się koszty utrzymania - zaznacza. - Miejsce to służy również do celów dydaktycznych. Widać stąd panoramę geologiczną Krakowa i okolic, w tym między innymi Dolinę Wisły, zrąb Wawelu i Sowińca, Płaskowyż Ojcowski czy Górę Pychowicką. 
Comme supra, en italique se démarquent les expressions adjacentes à écologie urbaine, ici on retrouve plutôt la collocation construction écologique. Les mots, expressions soulignés désignent les emplois concrets, tout ce qui est en corrélation avec la notion concrète de l'écologie'. Quant à l'emploi d'identification, il émerge grâce aux déterminants possessifs employés plusieurs fois.

L'écriture journalistique livre divers aspects de la notion écologique par le biais :

- des actions: donner un coup de neuf, pari sur l'avenir, gagner sur le long terme;

- de la dimension politiquement correct: technologie respectueuse de l'environnement, approche attentive aux besoins des habitants, l'avantage d'être plus respectueux de l'environnement;

- la dimension économique: aides financières, le taux zéro, intéressant économiquement, reprise du terme économique;

- les variations référentielles transparaissent grâce à la terminologie «environnementale».

Pour l'extrait polonais (d) sont mis en exergue en italique les pendants de écologie urbaine comme construction intelligente ${ }^{10}$. Les mots, expressions soulignés marquent les emplois concrets de l'idéologie de l'écologie. Pour l'emploi d'identification: czlowieka - l'homme - terme par excellence ressort, même s'il est précisé que son interférence est minimale. Quant aux aspects, ce sont les suivants qui émergent:

- les actions: Miejsce to stuży również do celów dydaktycznych - également en tant que lieu didactique;

- la dimension politiquement correct: ogrzewana cieptem Ziemi-chauffage par la chaleur de la Terre, instalacje fotowoltaiczna - installation photovoltaïque;

- la dimension économique: Zmniejsza się koszty - réduction des coûts, minimalizuje się koszty utrzymania - minimaliser les coûts d'entretien;

- les variations référentielles: inteligentne budownictwo - construction intelligente, Zielony dach - toit vert, ekologicznego wizerunku - image écologique.

Les discours recueillis, les collocations analysées ainsi que le dispositif sémiotique des quotidiens en ligne sont disparates. Nous nous en doutions, néanmoins, il nous semble que la terminologie employée en France se concentre plus sur le bien-être et les engagements plausibles, tandis que les énoncés polonais sont plus scientifiques et spécialisés.

9 Faute de place, nous ne reprenons pas les expressions dans le texte.

${ }^{10}$ Qui d'ailleurs est une collocation à l'occurrence de plus en plus fréquente (voir Martinez, 2018). «L'écologie urbaine est-elle si interprétable qu'elle le semble?», Terminologie(s) et traduction. Les termes de l'environnement et l'environnement des termes, Peter Lang, à paraître. 


\section{REMARQUES EN GUISE DE CONCLUSION}

Au cours de cette étude, nous avons vu que les faits sociaux se maintiennent grâce à une intentionnalité collective, c.-à-d. «le sentiment que l'on a de faire, vouloir, croire, etc. quelque chose ensemble» (Searle, 1998: 42). Que le sens social dans la mesure où l'individuation ${ }^{11}$ d'un événement relevait d'un "procès social qui mobilise des informations contextuelles, mais également des ressources symboliques, des croyances, des conventions sociales et intellectuelles, qui permettent de les interpréter sous une perspective intersubjective valide» (Barthélémy, 1992: 32).

$\mathrm{Au}$ cours des analyses, nous avons constaté que l'interaction entre les unités lexicales et les différents niveaux de la discursivité montre qu'un choix qui apparaît comme paradigmatique s'accompagne en réalité de choix syntagmatiques. Que la dualité du discours se scinde en face routinière - c.-à-d., un quotidien, répétitif, codé et l'usage, et en face «événementielle» singulière par l'acte d'énonciation d'un locuteur à un autre.

Pour conclure, nous constatons que l'élaboration du profil, si on le voit du point de vue de la production langagière, offre au locuteur des «prêt-à-parler», et que ceux-ci contribuent à la configuration du sens social. Nous pensons aussi que les habitus discursifs - français ou polonais, facilitent la transmission et l'acquisition du lexique et des messages.

\section{RÉFÉRENCES BIBLIOGRAPHIQUES}

Barthélémy, M. (1992). Événement et espaces publics : l'affaire Carpentras. Quaderni, 18, 125-140. Mika, M. (2007). Turystyka przyrodnicza - jej istota i współczesne kierunki rozwoju. Studia nad turystyka. Tradycje, stan obecny i perspektywy badawcze, 1, 311-320.

Moirand, S. (2004). L'impossible clôture des corpus médiatiques. La mise au jour des observables entre catégorisation et contextualisation. Tranel, 40, 71-92.

Moirand, S. (2007). Les discours de la presse quotidienne, observer, analyser, comprendre. Paris: PUF.

Plantin, C. (2003). Des polémiques aux polémiqueurs. In: G. Declercq, M. Murat, J. Dangel (éds.), La parole polémique (pp. 377-408). Paris : Honoré Champion.

Searle, J.-R. (1998). La construction de la réalité sociale. Paris: Gallimard.

Tutin, A., Grossmann, F. (2002). Collocations régulières et irrégulières: esquisse de typologie du phénomène collocatif. Revue française de linguistique appliquée, 7(1), 7-25.

Veniard, M. (2013). La nomination des événements dans la presse. Essai de sémantique discursive. Besançon: Presses universitaires de Franche-Comté.

${ }^{11}$ «Processus par lequel un groupe se caractérise face à un autre groupe grâce à des constances de l'activité langagière» (Ling, 1972; également dans Greimas-Courtés, 1979) CNRTL. 


\title{
RÉSUMÉ
}

Le lexique de l'écologie est un domaine très actuel qui développe de nombreux phrasèmes. Nous souhaitons présenter dans cet article le profil lexical (Veniard, 2013) de certaines collocations employées au quotidien dans les médias français. Nous proposons de voir la combinatoire ainsi que le fonctionnement discursif de certaines expressions lexicalisées pour pourvoir mettre en exergue les «traits sémiotiques» ressortant de notre analyse. Notre corpus est composé d'expressions figées ancrées dans les médias français que nous décortiquerons pour les comparer aux équivalents polonais. L'ambition de cette étude est de démontrer les buts discursifs divergents d'une culture à l'autre - d'un habitus discursif divergent dans l'emploi des expressions lexicalisées d'un domaine spécialisé.

Mots-clés : sème, profil lexico-discursif, collocation, contexte

\begin{abstract}
The aim of the article is to present some collocations as well as the profile of these lexicalised expressions commonly used in the French media. A semantic-discursive approach to some collocations (Sablayrolles, 2000, 2011; Sablayrolles \& Mejri, 2011; Tutin \& Grossmann, 2002; Tutin, 2005) allows us to create a lexical-discursive profile (Veniard, 2013). We will restrict ourselves to the semantic-pragmatic level of the profile (Veniard, 2013: 55). Bearing in mind that the profile is one of the aspects of discourse analysis, especially in French linguistics, and that it is immanent of French/francophone corpus, we will try to see if the profile of some collocations anchored in the French media have their equivalents in Polish.
\end{abstract}

Keywords: seme, lexical-discursive profile, collocation, context

\begin{abstract}
ABSTRAKT
Celem artykułu jest analiza niektórych kolokacji należących do pola leksykalnego écologie (ekologia) oraz opracowanie profilu utrwalonych wyrażeń, używanych powszechnie we francuskich mediach. Semantyczno-dyskursywny opis wymienionych połączeń (Sablayrolles, 2000, 2011; Sablayrolles, Mejri, 2011; Grossmann, Tutin, 2002; Tutin, 2005) pozwolił stworzyć ich profil leksykalno-dyskursywny (Veniard, 2013). W badaniach ograniczono się do poziomu semantyczno-pragmatycznego (Veniard, 2013: 55). Wiedząc, że profil jest jednym z możliwych aspektów analizy dyskursu, zwłaszcza w lingwistyce francuskiej, oraz jest immamentny dla korpusu języka francuskiego, starano się sprawdzić, czy profile analizowanych kolokacji w mediach francuskich mają odpowiedniki w języku polskim.
\end{abstract}

Słowa kluczowe: sem, dyskursywny profil leksykalny, kolokacja, kontekst 